\title{
Urethrovesikale Morphologie bei kontinenten Frauen in
} \section{Spinalanästhesie}

\begin{tabular}{|l|l|l|}
\hline E. & & Hanzal $^{\mathrm{a}}$ \\
\hline G. & & Häusler $^{\mathrm{a}}$ \\
\hline C. & Christine & Sam $^{\text {a }}$ \\
\hline H. & & Helmer $^{\mathrm{a}}$ \\
\hline H. & Astrid & Enzelsberger $^{\mathrm{a}}$ \\
\hline A. & & Chiari $^{\mathrm{b}}$ \\
\hline M. & & Gosch $^{\mathrm{b}}$ \\
\hline H. & Kölbl $^{\mathrm{a}}$ \\
\hline
\end{tabular}

${ }^{a}$ Universitätsklinik für Frauenheilkunde und ${ }^{b}$ Universitätsklinik für Anästhesie und allgemeine Intensivmedizin, Wien, Österreich

\section{Key Words}

Spinal anesthesia

Pelvic floor relaxation

Genuine stress incontinence

\section{Zusammenfassung}

Fragestellung: Wie wirkt sich eine neuromuskuläre Blockade des Beckenbodens bei kontinenten Frauen auf klinische, urodynamische und morphologische Befunde des unteren Harntraktes aus? Methoden: In einer prospektiven Studie wurden 14 kontinente Frauen ohne Deszensus unmittelbar vor und während Spinalanästhesie klinisch urodynamisch und sonographisch untersucht. Ergebnisse: Während der Spinalanästhesie zeigte sich gegenüber der präoperativen Evaluierung eine signifikant tiefere Blasenhalsposition und bei 4 von 7 Para ( 0 von 7 Nullipara) ein positiver klinischer Stresstest $(p=0,003)$. Schlussfolgerungen: Diese Ergebnisse unterstreichen die Bedeutung neuromuskulärer Faktoren für die Ätiologie von Beckenbodeninsuffizienz und Stressharninkontinenz. Dr. E. Hanzal, Abteilung für Gynäkologie und Geburtshilfe, Universitätsklinik für Frauenheilkunde, Spitalgasse 23, A-1090 Wien (Österreich)

Schlüsselwörter

Spinalanästhesie

Beckenbodeninsufñzienz

Stressharninkontinenz

Urethrovesical Morphology under Spinal Anesthesia in Continent Women

Objective: To assess the influence of spinal anesthesia on bladder neck position and a clinical stress test in continent women. Methods: In a prospective investigation, 14 women underwent urodynamic, sonographic and clinical assessment during spinal anesthesia. Results were compared to those obtained immediately preoperatively in the same patient. Results: During spinal anesthesia, the bladder neck was found to be located significantly lower and more posterior, and in 4/7 parous patients ( $0 / 7$ nullipara) the clinical stress test was positive. Conclusion: These data provide additional evidence for the importance of neuromuscular function in the etioloy of pelvic floor dysfunction and genuine stress incontinence. 
Morphologie urétro-vésicale de la femme continente sous anesthésie épidurale Objectif: Etude de $\Gamma$ effet, chez la femme continente, d'un blocage neuromusculaire du plan-cher pelvien sur les caractéristiques cliniques, urodynamiques et morphologiques des voies urinaires basses.

Méthodes: Dans le cadre d'une etude prospective, 14 femmes continentes ne présentant pas de descente d'organes ont subi un examen clinique, urodynamique et échogra-phique immédiatement avant et pendant une anesthésie épidurale. Résultats: L'évaluation pendant Tanesthésie épidurale a fait apparaître une position du col vésical significativement plus basse en comparaison de la période avant $\Gamma$ anesthésie et un test d'effort positif $(p=0,003)$ chez 4 sur 7 femmes ayant eu des enfants ( 0 sur 7 nullipares). Conclusions: Les résultats soulignent la signification des facteurs neuromusculaires dans Гétiologie des insufñsances pelviennes et de Tincontinence urinaire d'effort.

Einleitung

Für die morphologische Verankemng der Blasenhalsregion, de-ren Position für den Kontinenzmechanismus von entscheidender Bedeutung sein kann, sind sowohl bindegewebliche als auch musku-läre Bestandteile des Beckenbodens verantwortlich [1]. Eine Spinalanästhesie führt unter anderem auch zur annähernd kompletten

reversiblen Blockade der nervalen Versorgung der Beckenbodenmus-kulatur [2]. Es war das Ziel der vorliegenden prospektiven Untersu-chung zu überprüfen, ob eine Spinalanästhesie bei kontinenten Frauen zu einem sonographisch nachweisbaren Nachgeben des muskulä-ren Widerlagers im Blasenhalsbereich führt und ob dies das Ergebnis des klinischen Stresstestes beeinflusst.

C1995S. KargerAG, Basel

Tabelle 1. Sonographische Messgrössen vor und während Spinalanästhesie

$\mathrm{x}=$ Horizontalabstand $(\mathrm{mm}) ; \mathrm{y}-$ Vertikalabstand $(\mathrm{mm})$ des urethrovesikalen Überganges in bezug auf die Symphysenunterkan-te; $\beta=$ retrovesikaler Winkel (Grad).

1 Wilcoxon-Test.

Patientinnen und Methode

In die Studie wurden 14 Patientinnen (Alter: 33,1 Jahre; 19-53 Jahre), die sich kleineren gynäkologischen Eingriffen in Spinalanäst-hesie unterzogen (5 Hysteroskopien/fraktionierte Kürettagen, 6 Konisationen, 3 Laservaporisationen), aufgenommen. Auschlusskri-terien waren: neurologische Erkrankungen, degenerative Wirbelsäu-lenerkrankungen, Diabetes mellitus, vorangegangene vaginale Operationen oder Eingriffe im kleinen Becken, Harninkontinenz oder Deszensus. Die Hälfte der Frauen waren Nullipara, die anderen 7 Para mit 1-3 vaginalen Entbindungen. Von 2 Frauen wurden vaginal entbindende Operationen jeweils wegen protrahierter Geburt angegeben. Alle Frauen gaben nach ausführlicher Information ihr schriftliches Einverständnis für eine komplette urogynäkologische Untersuchung.

Die durchgeführten Tets bestanden aus Zystometrie, Urethroto-nometrie (Jupiter 8000; Wiest, Deutschland) [3], neurologischer Re-flexprüfung, klinischem Stresstest (bei $300 \mathrm{ml}$ Blasenvolumen) und einer Introitussonographie (5 MHz, 120 ${ }^{\circ}$, Vaginalsonde PVF $621 \mathrm{VT}$, Sonolayer SSA-250-1; Toshiba Corp., Japan) auf dem Opera-tionstisch unmittelbar präoperativ und ein zweites Mai während der Spinalanästhesie vor dem Eingriff. Die Methodik der Introitussono- 
graphie wurde anderswo bereits ausführlich beschrieben [4] und für diese Studie bei einer Blasenfüllung von $300 \mathrm{ml}$ durchgeführt, wobei die Lokalisation des Blasenhalses mit Hilfe eines $\mathrm{x}, \mathrm{y}$-Koordinatensy-stems in Relation zur unteren Symphysenkante ermittelt wurde.

Die Spinalanästhesie erfolgte generall ohne Prämedikation. Dazu wurde der Spinalkanal mit einer 27-G-Whittaker-Nadel zwischen L3 und L4 punktiert und 1,5 $\mathrm{ml}$ 5\% hyperbares Lidocain intrathekal appliziert. Vor der zweiten urogynäkologischen Untersuchung wurde bei alien Probandinnen ein Analgesieniveau von Thg verifíziert. Alle Untersuchungen wurden in Steinschnittlage bei exakt horizontalem Rücken durchgeführt. Der Wilcoxon-Test bzw. Fisherexact-Test diente zur statistischen Auswertung.

Ergebnisse

Alle Patientinnen zeigten präoperativ normale Untersuchungser-gebnisse, und alle waren im Rahmen des klinischen Stresstestes kon-tinent. Bei liegender Spinalanästhesie war der Blasenhals gegenüber präoperativ signifikant tiefer und weiter hinter der Symphyse lokali-siert. Der retrovesikale Winkel (B) war sowohl in Ruhe als auch beim Pressversuch während Spinalanästhesie signifikant grosser (Tab. 1). Alle Nullipara zeigten auch während Spinalanästhesie einen negati-ven klinischen Stresstest. Demgegenüber war bei 4 von 7 Para ein positiver klinischer Stresstest zu verzeichnen (Fisher-exact-Test: $p=0,003$ ).

Diskussion

Die vaginale Entbindung ist als Risikofaktor für die Beckenbo-deninsuffizienz und Stressharninkontinenz schon lange bekannt. Ne-ben den direkten Auswirkungen auf die muskulären Anteile des Bek-kenbodens werden hauptsächlich Schädigungen des Nervus pudendus als ausschlaggebend angesehen [5,6]. Diese Untersuchung zeigt, dass es bei einer Blockade der nervalen Versorgung der Beckenbo-denmuskulatur zu einem signifikanten Tiefertreten der Blasenhalsre-gion kommt. Bei 4/7 Frauen nach vaginalen Entbindungen kam es ausserdem zum Harnabgang beim klinischen Stresstest während der Spinalanästhesie. Dies unterstreicht die ätiologische Bedeutung der vaginalen Entbindung für die Stressharninkontinenz.

Weitere Untersuchungen sind notwendig, um zu klären, ob durch einen klinischen Stresstest während Spinalanästhesie eine Gruppe von Patientinnen mit höherem Risiko für eine später auftretende Stressinkontinenz identifizierbar ist und ob diese Frauen von pro-phylaktischen Massnahmen profítieren könnten.

\section{Literatur}

Hanzal E, Kölbl H: Abdominal, vaginal oder kombiniert: Verschiedene Wege zum gemeinsamen Ziel? Gynäkol Geburtshilfliche Rundschl992;33:51.

Axelsson K, Möllefors K, Olsson JO, Lind-gardh G, Widman B: Bladder function in spinal anesthesia. Acta Anaesthesiol Scand 1985; 29:315.

Hanzal E, Berger E, Koelbl H: Reliability of the urethral closure pressure profile during stress in the diagnosis of genuine stress incontinence. Br JUrol 1991:68:369.

Koelbl H, Hanzal E, Bernaschek G: Sono-graphic urethrocystography - Methods and application in patients with genuine stress incontinence. Int Urogynecol J 1991;2:25. Smith A, Hosker G, Warrell D: The role of pudendal nerve damage in the etiology of genuine stress incontinence in women. Br J Ob-stetGynaecoll989;96:29. Tapp A, Cardozo L, Versi E, Montgomery J, Studd J: The effect of vaginal delivery on the urethral sphincter. Br J Obstet Gynaecol 1988; 95:142.

174

Hanzal/Häusler/Sam/Helmer/Enzelsberger/ Chiari/Gosch/Kölbl 
Urethrovesikale Morphologie bei kontinenten Frauen in Spinalanästhesie 